\title{
Isoperimetric Numbers of Regular Graphs of High Degree with Applications to Arithmetic Riemann Surfaces
}

\author{
Dominic Lanphier* \\ Department of Mathematics \\ Western Kentucky University \\ Bowling Green, KY 42101, U.S.A. \\ dominic.lanphier@wku.edu
}

\author{
Jason Rosenhouse \\ Department of Mathematics and Statistics \\ James Madison University \\ Harrisonburg, VA 22807, U.S.A. \\ rosenhjd@jmu.edu
}

Submitted: Feb 7, 2011; Accepted: Jul 21, 2011; Published: Aug 12, 2011

Mathematics Subject Classifications: 05C40, 30F10

\begin{abstract}
We derive upper and lower bounds on the isoperimetric numbers and bisection widths of a large class of regular graphs of high degree. Our methods are combinatorial and do not require a knowledge of the eigenvalue spectrum. We apply these bounds to random regular graphs of high degree and the Platonic graphs over the rings $\mathbb{Z}_{n}$. In the latter case we show that these graphs are generally nonRamanujan for composite $n$ and we also give sharp asymptotic bounds for the isoperimetric numbers. We conclude by giving bounds on the Cheeger constants of arithmetic Riemann surfaces. For a large class of these surfaces these bounds are an improvement over the known asymptotic bounds.
\end{abstract}

\section{Introduction}

Let $G$ be a graph and let $A \subseteq V(G)$. The boundary of $A$, denoted by $\partial A$, is the set of edges of $G$ having precisely one endpoint in $A$. The isoperimetric number of $G$ is

$$
h(G)=\inf _{A} \frac{|\partial A|}{|A|}
$$

where the infimum is taken over all subsets $A \subset V(G)$ satisfying $|A| \leq \frac{1}{2}|V(G)|$. The isoperimetric number of a graph was introduced by Buser in [4] as a discrete analog of

${ }^{*}$ The author is partially supported by grant \# 223120 of Western Kentucky University 
the Cheeger constant used to study the eigenvalue spectrum of a Riemannian manifold. The bisection width $b w(G)$ is $\inf _{A}|\partial A|$ where $n-2|A| \leq 1$.

For a regular graph of degree $k$, it is now standard to estimate $h(G)$ in terms of the second largest eigenvalue of the adjacency matrix of $G$ as in [7], [16] and [17]. This approach is especially suited to Cayley graphs (and quotients of Cayley graphs) of groups whose character tables are readily determined, as in [16]. In these cases one can obtain spectral information about the graph following the representation theoretic methods of [2]. However, this method is more difficult for Cayley graphs of groups whose representations are less tractable. Recently, combinatorial and elementary methods have been used to construct explicit families of expanders as in [1] and [19]. In this paper we use combinatorial methods to obtain upper and lower bounds on the isoperimetric number for large classes of regular graphs. We then give applications to random regular graphs of high degree and to the Platonic graphs. We use the latter results to study the Cheeger constants of arithmetic Riemann surfaces.

Our main results are Theorems 1 and 3 and Corollary 2 below. We show that for a highly connected regular graph, specifically any graph in which an arbitrary vertex is connected by a 2-path to at least half of the other vertices, we can derive upper and lower bounds for the isoperimetric number. From Corollary 1 we see that these estimates are asymptotically sharp for most graphs of high degree.

Theorem 1. Let $G$ be a k-regular graph with $|V(G)|=n$. Assume that for any $v \in V(G)$ there are at least $r$ paths of length 2 from $v$ to every vertex in a set of size $n-m$, where $0 \leq m \leq n / 2$ and $m$ does not depend on $v$. Also assume that $k^{2} \geq r(n-m)$. Then
i) $\frac{1}{2}\left(k+\sqrt{k^{2}-r(n-2 m)}\right) \geq h(G) \geq \frac{1}{2}\left(k-\sqrt{k^{2}-r(n-2 m)}\right)$
ii) $\frac{n}{4}\left(k+\sqrt{k^{2}-r\left(n-\frac{4 m^{2}}{n}\right)}\right) \geq b w(G) \geq \frac{n}{4}\left(k-\sqrt{k^{2}-r\left(n-\frac{4 m^{2}}{n}\right)}\right)$.

Note that in the case of a graph $G$ with the properties that $m=0, r=1$ and $k=\sqrt{n}$ (exactly) then we have the exact values $h(G)=k / 2$ and $b w(G)=k n / 4$.

We apply Theorem 1 to two classes of graphs: random regular graphs of high degree as in [11], and Platonic graphs as in [8], [9], [13], and [15]. This gives Corollary 1 and Theorem 3.

The model $G_{n, k}$ of random regular graphs consists of all regular graphs of degree $k$ on $n$ vertices with the uniform probability distribution. As in [3] we use $G_{n, k}$ to denote both the probability space and a random graph in the space.

We say that a statement depending on $n$ occurs almost always asymptotically (a.a.s.) if the statement occurs with probability approaching 1 as $n$ goes to $\infty$.

Corollary 1. Let $\omega(n)$ denote any function that grows arbitrarily slowly to $\infty$ with $n$. Suppose that $k^{2}>\omega(n) n \log (n)$ and $k \in o(n)$. Then a.a.s.

$$
\frac{k}{2}\left(1+O\left(\frac{1}{n}\right)\right) \geq h\left(G_{n, k}\right) \geq \frac{k}{2}\left(1-O\left(\frac{1}{\sqrt{\omega(n)}}\right)\right) .
$$


Note that this is essentially Corollary 2.10 in [11].

Recall that a $k$-regular graph $G$ is called Ramanujan if for all eigenvalues $\lambda$ of the adjacency operator where $|\lambda| \neq k$ we have $|\lambda| \leq 2 \sqrt{k-1}$. In the sequel we let $\lambda_{1}$ denote the largest eigenvalue less than $k$.

Let $R$ be a finite commutative ring with identity and define

$$
\mathcal{S}_{R}=\left\{(\alpha, \beta) \in R^{2} \mid \text { there exist } x, y \in R \text { such that } a x-b y=1\right\} .
$$

The Platonic graphs $\pi_{R}$ are defined by $V\left(\pi_{R}\right)=\left\{(\alpha, \beta) \in R^{2} \mid(\alpha, \beta) \in \mathcal{S}_{R}\right\}$ and $(\alpha, \beta)$ is adjacent to $(\gamma, \delta)$ if and only if $\operatorname{det}\left(\begin{array}{cc}\alpha & \beta \\ \gamma & \delta\end{array}\right)= \pm 1$. These graphs have been well-studied and are related to the geometry of modular surfaces [5], [6], [13]. Further, for certain rings $R$ the Platonic graphs $\pi_{R}$ provide examples of elementary Ramanujan graphs as in [9]. In particular, for $\mathbb{F}_{q}$ the finite field with $q$ elements we have the following:

Theorem $2([8,9,15])$. Let $p$ be an odd prime and let $q=p^{r}$. Then $\pi_{\mathbb{F}_{q}}$ is Ramanujan.

This was proved by determining the spectrum of these graphs from the character table of $G L_{2}\left(\mathbb{F}_{q}\right)$ as in [16]. The character table of $G L_{2}(R)$ for $R=\mathbb{F}_{q}$ is well-known, see [18] for example. For other rings, in particular for $R=\mathbb{Z}_{N}$ with $N$ composite, the representations of $G L_{2}(R)$ and $S L_{2}(R)$ are more complicated. See [12] for a study of the characters of $S L_{2}\left(\mathbb{Z}_{p^{n}}\right)$, for example.

Although the graphs $\pi_{\mathbb{Z}_{N}}$ form families of expanders [13], it is expected that they are generally not Ramanujan for composite $N$. Further, as presented in the discussion at the end of Section 4 in [9], it is not known precisely which $\pi_{\mathbb{Z}_{N}}$ are Ramanujan. It is noted there that $\pi_{\mathbb{Z}_{N}}$ is not Ramanujan for $N=p q$ with $q$ sufficiently larger than $p$.

In the following we give upper and lower bounds of the same order for $h\left(\pi_{\mathbb{Z}_{N}}\right)$. We apply Theorem 1 to give lower bounds on certain $h\left(\pi_{\mathbb{Z}_{N}}\right)$ of the same order as the upper bounds. Then we show that in general the graphs $\pi_{\mathbb{Z}_{N}}$ are not Ramanujan.

Theorem 3. i) For odd, composite $N$ we have

$$
\frac{N}{2}-\frac{1}{\prod_{p \mid N}\left(1+\frac{1}{p}\right)} \geq h\left(\pi_{\mathbb{Z}_{N}}\right) \geq \frac{N}{2}\left(1-\sqrt{1-2 \prod_{p \mid N}\left(1-\frac{1}{p}\right)+\prod_{p \mid N}\left(1-\frac{1}{p^{2}}\right)}\right) .
$$

Thus for any $\epsilon>0$ and sufficiently large $N$ with $\prod_{p \mid N}\left(1+\frac{1}{p}\right)$ sufficiently close to 1 we have

$$
\frac{N}{2}-1+\epsilon \geq h\left(\pi_{\mathbb{Z}_{N}}\right) \geq \frac{N}{2}(1-\epsilon) .
$$

ii) For odd, composite $N$ with $\prod_{p \mid N}\left(1+\frac{1}{p}\right)$ sufficiently large we have $h\left(\pi_{\mathbb{Z}_{N}}\right) \leq c N$ for some $c<1 / 2$. Thus, for such $N, \pi_{\mathbb{Z}_{N}}$ is not Ramanujan.

We can also obtain estimates on the bisection width of $\pi_{\mathbb{Z}_{N}}$ using (ii) of Theorem 1 . Note that the upper bound in $(i)$ of Theorem 3 was first shown for primes $p \equiv 1(\bmod 4)$ in [5] and extended to odd prime powers in [13]. 
Recall that the group $\Gamma_{N}=P S L_{2}\left(\mathbb{Z}_{N}\right)$ acts on the complex upper half plane $\mathcal{H}$ via linear fractional transformations. Let $\Gamma_{N} \backslash \mathcal{H}$ denote a fundamental domain for this action. The Cheeger constants $h\left(\Gamma_{N} \backslash \mathcal{H}\right)$ of these surfaces have been well-studied [4], [5], and [6]. Precise definitions of these surfaces and their Cheeger constants are given in Section 5 . Using probabilistic methods, Brooks and Zuk in [6] showed that $h\left(\Gamma_{N} \backslash \mathcal{H}\right) \leq 0.4402$ for sufficiently large $N$. From (i) of Theorem 3 and inequality (12) in Section 4 we have a sharper bound for the cases $N=3,3^{2}$, and $5^{r}$. Further, we have:

Corollary 2. For sufficiently large odd composite $N$ with $\prod_{p \mid N}\left(1+\frac{1}{p}\right)$ sufficiently large,

$$
h\left(\Gamma_{N} \backslash \mathcal{H}\right) \leq A
$$

where $A<0.4402$ can be given explicitly and depends on $N$.

In Section 2 we prove Theorem 1 and use a result from [11] to give a new proof of Corollary 1. In Section 3 we show that the Platonic graphs are isomorphic to certain quotients of Cayley graphs of $P S L_{2}(R)$. This allows us to apply counting arguments to $\pi_{R}$. In Section 4 we prove Theorem 3 and investigate the asymptotic properties of $h\left(\pi_{\mathbb{Z}_{N}}\right)$. Finally, in Section 5 we discuss the arithmetic Riemann surfaces under consideration and prove Corollary 2.

\section{Proof of Theorem 1}

Let $G$ be a simple regular graph of degree $k$ and let $|V(G)|=n$. Let $A \subset V(G)$ with $|A| \leq n / 2$ and let $B=V(G) \backslash A$. Let $\partial A$ denote the boundary of $A$. For $v \in A$ define

$$
\partial v=\{e \in \partial A \mid e \text { is incident with } v\} .
$$

Note that $|\partial A|=\sum_{v \in A}|\partial v|$.

Let $e \in \partial A$ with $e=\left(v_{e}, w_{e}\right)$ where $v_{e} \in A$ and $w_{e} \in B$. Let

$$
\begin{aligned}
& \partial_{A} e=\left\{e^{\prime} \in \partial A \mid e^{\prime} \text { incident with } v_{e}\right\}, \\
& \partial_{B} e=\left\{e^{\prime} \in \partial A \mid e^{\prime} \text { incident with } w_{e}\right\} .
\end{aligned}
$$

Note that in any path of length 2 having one endpoint in $A$ and one endpoint in $B$, it must be the case that one of the edges is in $\partial A$ (equivalently $\partial B$ ), while the other edge either has both endpoints in $A$ or both endpoints in $B$. When the non-boundary edge lies entirely within $A$ we shall say that the path "begins in $A$," otherwise the path will be said to "begin in $B . "$

Let $e \in \partial A$ be in a path of length 2 from $A$ to $B$. Let $e=(v, w)$ with $v \in A$ and $w \in B$. If $v$ is the midpoint of a path of length 2 then the path must begin in $A$, as otherwise it would begin and end in $B$. Thus there are $k-\left|\partial_{A} e\right|$ choices for the beginning vertex of the path. Similarly, if $w$ is the midpoint, then there are $k-\left|\partial_{B} e\right|$ choices for the endpoint of the path. Therefore, an edge $e \in \partial A$ from $v \in A$ to $w \in B$ lies in

$$
\left(k-\left|\partial_{A} e\right|\right)+\left(k-\left|\partial_{B} e\right|\right)=2 k-\left|\partial_{A} e\right|-\left|\partial_{B} e\right|
$$


paths of length 2 from $A$ to $B$. It follows that there are no more than $\sum_{e \in \partial A} 2 k-\left|\partial_{A} e\right|-$ $\left|\partial_{B} e\right|$ paths of length 2 from $A$ to $B$. By hypothesis, there are at least $r$ paths of length 2 from any $v \in A$ to a subset of $B$ of size $|B|-m$, where $m$ does not depend on $v$. Thus there exist (at least) $r|A|(|B|-m)$ paths of length 2 connecting $A$ to $B$. It follows that

$$
\sum_{e \in \partial A} 2 k-\left|\partial_{A} e\right|-\left|\partial_{B} e\right| \geq r|A|(|B|-m) .
$$

Note that

$$
\begin{aligned}
\sum_{e \in \partial A}\left|\partial_{A} e\right|=\sum_{v \in A} \sum_{\substack{e \in \partial A \\
e \text { incident with } v}}\left|\partial_{A} e\right| & =\sum_{v \in A} \sum_{\substack{e \in \partial A \\
e \text { incient with } v}}|\partial v| \\
& =\sum_{v \in A}|\partial v| \sum_{\substack{e \in \partial A \\
e \text { incident with } v}} 1=\sum_{v \in A}|\partial v|^{2}
\end{aligned}
$$

and $\sum_{e \in \partial A}\left|\partial_{B} e\right|=\sum_{e \in \partial B}\left|\partial_{B} e\right|$.

Let $t=|\partial A| /|A|, a=|A|$, and $b=|B|$. By the Cauchy-Schwartz inequality, $|A| \sum_{v \in A}|\partial v|^{2} \geq|\partial A|^{2}$ and so $\sum_{e \in \partial A}\left|\partial_{A} e\right| \geq a t^{2}$. Thus (1) gives

$$
r(b-m) \leq \frac{1}{a} \sum_{e \in \partial A} 2 k-\left|\partial_{A} e\right|-\left|\partial_{B} e\right| \leq 2 k t-t^{2}-t^{2} \frac{a}{b}=2 k t-t^{2}\left(1+\frac{a}{b}\right) .
$$

Now, $2 k-t\left(1+\frac{a}{b}\right)>0$. To see this assume otherwise and note that $t<k$. Since $a \leq n / 2$ we have $b \geq n / 2$. It follows that $2 k \leq t n / b<k n / b \leq 2 k$ which gives a contradiction. As $k^{2} \geq r(n-m)$ we can apply the quadratic formula to get

$$
\frac{b}{n}\left(k+\sqrt{k^{2}-n r\left(1-\frac{m}{b}\right)}\right) \geq t \geq \frac{b}{n}\left(k-\sqrt{k^{2}-n r\left(1-\frac{m}{b}\right)}\right) .
$$

This holds for $0<a \leq n / 2$ and so for all $n>b \geq n / 2$. Define

$$
f(x)=\frac{n-x}{n}\left(k-\sqrt{k^{2}-n r\left(1-\frac{m}{n-x}\right)}\right) .
$$

Then

$$
f^{\prime}(x)=-\frac{1}{n}\left(k-\sqrt{k^{2}-n r\left(1-\frac{m}{n-x}\right)}\right)-\left(\frac{n-x}{n}\right) \frac{1}{2 \sqrt{k^{2}-n r\left(1-\frac{m}{n-x}\right)}} \frac{m}{(n-x)^{2}}
$$

which is less than 0 for $n>x>0$. Thus $f(x)$ is decreasing and as $n>n-x=b \geq n / 2$ then $n / 2 \geq x>0$ and so the right hand side of (2) is maximal at $x=n / 2$. This gives the lower bound from (i) of Theorem 1 . Note that similar, but significantly weaker, lower 
bounds on the isoperimetric constant were found in [14]. Since $h(G)$ is an infimum we have from (2) that

$$
\frac{b}{n}\left(k+\sqrt{k^{2}-r n\left(1-\frac{m}{b}\right)}\right) \geq t \geq h(G)
$$

for any $n>b \geq n / 2$. Taking $b=n / 2$ gives the upper bound, and this completes the proof of (i) of Theorem 1 .

In the case where the isoperimetric set satisfies $n-2 a \leq 1$ we have $a \geq m$. We can count the 2-paths from $m$ remaining vertices in $B$ to $a-m$ vertices in $A$. Thus there exist at least $r a(b-m)+r m(a-m)=r\left(a n-m^{2}\right) 2$-paths from $A$ to $B$. Applying the same analysis as above we get

$$
\frac{b}{n}\left(k+\sqrt{k^{2}-n r\left(1-\frac{m^{2}}{a b}\right)}\right) \geq t \geq \frac{b}{n}\left(k-\sqrt{k^{2}-n r\left(1-\frac{m^{2}}{a b}\right)}\right) .
$$

This completes the proof of Theorem 1.

To prove Corollary 1, we recall the main result from [11]. For $v \in V(G)$ let $N(v)$ denote the set of vertices adjacent to $v$. Then $\operatorname{codeg}(u, v)=|N(u) \cap N(v)|$. Recall that a set of graphs $A_{n}$ are a.a.s. in the space $G_{n, k}$ if $\lim _{n \rightarrow \infty} P\left(A_{n}\right)=1$.

Theorem 4 (Theorem 2.1, [11]). Let $\omega(n)$ denote any function that grows arbitrarily slowly to $\infty$ with $n$. Suppose that $k^{2}>\omega(n) n \log (n)$.

(i) If $k<n-c n / \log (n)$ for some $c>2 / 3$ then a.a.s.

$$
\max _{u, v}\left|\operatorname{codeg}(u, v)-\frac{k^{2}}{n}\right|<C \frac{k^{3}}{n^{2}}+6 \frac{k \sqrt{\log (n)}}{\sqrt{n}}
$$

where $C$ is an absolute constant.

(ii) If $k \geq c n / \log (n)$ then a.a.s.

$$
\max _{u, v}\left|\operatorname{codeg}(u, v)-\frac{k^{2}}{n}\right|<6 \frac{k \sqrt{\log (n)}}{\sqrt{n}} .
$$

(iii) If $3 \leq k=O\left(n^{1-\delta}\right)$ then $\operatorname{codeg}(u, v)<\max \left(k^{1-\epsilon(\delta)}, 3\right)$.

It follows that for sufficiently large $n$ and for $k^{2}>\omega(n) n \log (n)$, the number of paths of length 2 from $u$ to $v$ is a.a.s. greater than or equal to

$$
\frac{k^{2}}{n}-\left(C \frac{k^{3}}{n^{2}}+6 \frac{k \sqrt{\log (n)}}{\sqrt{n}}\right) \text {. }
$$

Note that since $k \in o(n)$ the above expression is greater than 0 , and in fact grows arbi- 
trarily large with $n$. From (i) of Theorem 1, we have that a.a.s.,

$$
\begin{aligned}
h\left(G_{n, k}\right) & \geq \frac{1}{2}\left(k-\sqrt{k^{2}-\left(\frac{k^{2}}{n}-C \frac{k^{3}}{n^{2}}-6 \frac{k \sqrt{\log (n)}}{\sqrt{n}}\right) n}\right) \\
& =\frac{1}{2}\left(k-k \sqrt{C \frac{k}{n}+6 \frac{\sqrt{n \log (n)}}{k}}\right) \\
& =\frac{k}{2}\left(1-O\left(\sqrt{\frac{\sqrt{n \log (n)}}{k}}\right)\right) .
\end{aligned}
$$

The upper bound from Corollary 1 derives from random methods and is well-known.

\section{Quotients of Cayley Graphs of Matrix Groups}

To study the Platonic graphs $\pi_{R}$ for a finite commutative ring $R$ with identity, we show how to express them as quotients of Cayley graphs of $P S L_{2}(R)$. This allows us to determine explicit formulas for the orders of $\pi_{R}$ for certain $R$, as well as related quantities.

Let $\Gamma$ be a finite group and let $S$ be a generating set for $\Gamma$. If $S=S^{-1}$ then we say that $S$ is symmetric. The Cayley graph of $\Gamma$ with respect to the symmetric generating set $S$, denoted $G(\Gamma, S)$, is defined as follows: The vertices of $G$ are the elements of $\Gamma$. Distinct vertices $\gamma_{1}$ and $\gamma_{2}$ are adjacent if and only if $\gamma_{1}=\omega \gamma_{2}$ for some $\omega \in S$. Cayley graphs are $|S|$-regular. Since the permutation of the vertices induced by right multiplication by a group element is easily shown to be a graph automorphism, it follows that Cayley graphs are vertex-transitive. If $g_{1}$ and $g_{2}$ are adjacent vertices in a Cayley graph, then we will write $g_{1} \sim g_{2}$

Let $R$ be a finite commutative ring with identity and let $R^{\times}$be the group of units of $R$. Let

$$
\Gamma_{R}=P S L_{2}(R)=\left\{\left(\begin{array}{ll}
a & b \\
c & d
\end{array}\right) \mid a d-b c=1\right\} /\langle \pm 1\rangle
$$

Set

$$
N_{R}=\left\{\left(\begin{array}{ll}
1 & x \\
0 & 1
\end{array}\right) \mid x \in R\right\}
$$

and let $Z(R)$ denote the semigroup of zero divisors of $R$.

Let $\omega \in R^{\times}$and let $S_{R}$ be a symmetric generating set for $\Gamma_{R}$ containing $\left(\begin{array}{cc}0 & \omega \\ -\omega^{-1} & 0\end{array}\right) \in S_{R}$, with all other $\xi \in S_{R}$ in $N_{R}$. Let $G_{R}=G\left(\Gamma_{R}, S_{R}\right)$ denote the corresponding Cayley graph.

If $g$ is any element in $\Gamma_{R}$ then left multiplication by elements of $N_{R}$ does not change the bottom row of $g$. It follows that elements of $\Gamma_{R}^{\prime}=N_{R} \backslash \Gamma_{R}$ can be indexed by

$$
\Gamma_{R}^{\prime} \cong\left\{(\alpha, \beta) \mid \alpha, \beta \in R, \quad(\alpha, \beta) \notin Z(R)^{2}\right\} /\langle \pm 1\rangle .
$$


Consider the quotient graph $G_{R}^{\prime}=N_{R} \backslash G_{R}$ (i.e. the multigraph whose vertices are given by the cosets in $\Gamma_{R}^{\prime}$, with distinct cosets $N_{R} \gamma_{1}$ and $N_{R} \gamma_{2}$ joined by as many edges as there are edges in $G_{R}$ of the form $\left(v_{1}, v_{2}\right)$, where $v_{1} \in N_{R} \gamma_{1}$ and $\left.v_{2} \in N_{R} \gamma_{2}\right)$. Since $\Gamma_{R}^{\prime}$ is not a group $\left(N_{R}\right.$ is not normal in $\left.\Gamma_{R}\right)$, these quotient graphs are not themselves Cayley graphs. They are, however, induced from the Cayley graph $G_{R}$. In the sequel we make no distinction between a vertex in our graph and the group element it represents.

Lemma 1. Let $(\alpha, \beta)$ and $(\gamma, \delta)$ be vertices in $G_{R}^{\prime}$. Then $(\alpha, \beta) \sim(\gamma, \delta)$ if and only if

$$
\operatorname{det}\left(\begin{array}{ll}
\alpha & \beta \\
\gamma & \delta
\end{array}\right)= \pm \omega, \pm \omega^{-1}
$$

Proof. Let $g \in V\left(G_{R}\right)$. Left multiplication of $g$ by elements of $N_{R}$ preserves the bottom row of $g$. Therefore, $g^{\prime} \in G_{R}^{\prime}$ is adjacent to precisely those elements attainable from it by left multiplication by $\xi \in S_{R}$, with $\xi \notin N_{R}$. Observe that $\left(\begin{array}{cc}0 & \omega \\ -\omega^{-1} & 0\end{array}\right)\left(\begin{array}{ll}a & b \\ c & d\end{array}\right)=\left(\begin{array}{cc}\omega c & \omega d \\ -\omega^{-1} a-\omega^{-1} b\end{array}\right)$. Thus if $(\alpha, \beta) \sim(\gamma, \delta)$ then we must have $\operatorname{det}\left(\begin{array}{cc}\alpha & \beta \\ \gamma & \delta\end{array}\right)= \pm \omega, \pm \omega^{-1}$ as was to be shown.

For the reverse direction, note that if $\alpha \delta-\beta \gamma= \pm \omega, \pm \omega^{-1}$, then we must have that $\left(\begin{array}{cc}\epsilon \alpha & \epsilon \beta \\ \gamma & \delta\end{array}\right) \in \Gamma_{R}$ for some $\epsilon \in\left\{ \pm \omega, \pm \omega^{-1}\right\}$. But then it is clear that left multiplication by an element of $S_{R}-N_{R}$ will take $(\alpha, \beta)$ to $\epsilon^{\prime}(\gamma, \delta)$ with $\epsilon^{\prime} \in\left\{ \pm \omega, \pm \omega^{-1}\right\}$ and the proof is complete.

As a consequence we see that if $\omega= \pm 1$ then $\pi_{R}$ is isomorphic to $G_{R}^{\prime}$.

Lemma 2. Let $(\alpha, \beta),\left(\alpha^{\prime}, \beta^{\prime}\right) \in V\left(G_{R}^{\prime}\right)$ satisfy $\operatorname{det}\left(\begin{array}{cc}\alpha & \beta \\ \alpha^{\prime} & \beta^{\prime}\end{array}\right) \in R^{\times}$. If $\omega^{2}=1$ (resp. $\left.\neq 1\right)$ then there are exactly 2 (resp. 4) paths of length 2 joining $(\alpha, \beta)$ to $\left(\alpha^{\prime}, \beta^{\prime}\right)$.

Proof. From Lemma 1, a path of length 2 joining $(\alpha, \beta)$ to $\left(\alpha^{\prime}, \beta^{\prime}\right)$ is given by a vector $(\gamma, \delta)$ such that $\operatorname{det}\left(\begin{array}{cc}\alpha & \beta \\ \gamma & \delta\end{array}\right) \equiv \pm \omega, \pm \omega^{-1}$ and $\operatorname{det}\left(\begin{array}{cc}\gamma & \delta \\ \alpha^{\prime} & \beta^{\prime}\end{array}\right) \equiv \pm \omega, \pm \omega^{-1}$. Set $\xi=\operatorname{det}\left(\begin{array}{cc}\alpha & \beta \\ \alpha^{\prime} & \beta^{\prime}\end{array}\right) \in R^{\times}$. By elementary linear algebra, there are nonzero elements $c_{1}, c_{2} \in R$ so that $(\gamma, \delta)=$ $c_{1}(\alpha, \beta)+c_{2}\left(\alpha^{\prime}, \beta^{\prime}\right)$. A straightforward computation shows that

$$
\operatorname{det}\left(\begin{array}{ll}
\alpha & \beta \\
\gamma & \delta
\end{array}\right)=c_{2} \operatorname{det}\left(\begin{array}{cc}
\alpha & \beta \\
\alpha^{\prime} & \beta^{\prime}
\end{array}\right)=c_{2} \xi
$$

and

$$
\operatorname{det}\left(\begin{array}{cc}
\gamma & \delta \\
\alpha^{\prime} & \beta^{\prime}
\end{array}\right)=c_{1} \operatorname{det}\left(\begin{array}{cc}
\alpha & \beta \\
\alpha^{\prime} & \beta^{\prime}
\end{array}\right)=c_{1} \xi
$$

This leads to 4 or 8 ordered pairs $\left(c_{1}, c_{2}\right)$ for which the vector $(\gamma, \delta)$ has the desired properties. Since vectors differing only by a factor of -1 are identical, these pairs represent 2 or 4 distinct paths in $G_{R}^{\prime}$.

Lemma 3. Let $(\alpha, \beta) \in \Gamma_{R}^{\prime}$, then

$$
\#\left\{\left(\alpha^{\prime}, \beta^{\prime}\right) \in \Gamma_{R}^{\prime} \mid \operatorname{det}\left(\begin{array}{cc}
\alpha & \beta \\
\alpha^{\prime} & \beta^{\prime}
\end{array}\right) \in R^{\times}\right\}=\frac{|R|\left|R^{\times}\right|}{2} .
$$


Proof. If $\alpha^{\prime}, \beta^{\prime} \in Z(R)$ then there is some nonzero $z \in Z(R)$ so that $z \alpha^{\prime}=z \beta^{\prime}=0$. It follows that if $\alpha \beta^{\prime}-\beta \alpha^{\prime} \in R^{\times}$then one of $\alpha^{\prime}$ or $\beta^{\prime}$ cannot be in $Z(R)$ and so $\left(\alpha^{\prime}, \beta^{\prime}\right) \in \Gamma_{R}^{\prime}$.

First we count the number of $\left(\alpha^{\prime}, \beta^{\prime}\right)$ so that $\alpha \beta^{\prime}-\beta \alpha^{\prime}=1$. If $\alpha \in R^{\times}$then $\left(\alpha^{\prime}, \beta^{\prime}\right)=$ $\left(\alpha^{-1}\left(1+\beta \beta^{\prime}\right), \beta^{\prime}\right)$ works and if $\beta \in R^{\times}$then $\left(\alpha^{\prime}, \beta^{-1}\left(\alpha \alpha^{\prime}-1\right)\right)$ works for any $\beta^{\prime}$ (resp. $\left.\alpha^{\prime}\right)$ in $R$. Thus there are $|R|$ possible choices of $\left(\alpha^{\prime}, \beta^{\prime}\right) \in \Gamma_{R}^{\prime}$ so that $\operatorname{det}\left(\begin{array}{c}\alpha \\ \alpha^{\prime}\end{array} \beta^{\prime}\right)=1$. For each such choice, there are $\left|R^{\times}\right|$further choices for $\operatorname{det}\left(\begin{array}{cc}\alpha & \beta \\ \alpha^{\prime} & \beta^{\prime}\end{array}\right) \in R^{\times}$. This gives the result.

\section{Applications to Platonic Graphs}

Set $R=\mathbb{Z}_{N}, U=\left(\begin{array}{ll}1 & 1 \\ 0 & 1\end{array}\right)$ and $V=\left(\begin{array}{cc}0 & 1 \\ -1 & 0\end{array}\right)$. Then $S_{N}=\left\{U, U^{-1}, V\right\}$ is a symmetric generating set for $\Gamma_{N}=P S L_{2}\left(\mathbb{Z}_{N}\right)$ satisfying the requirements of the previous section [13]. Following that notation, define $G_{N}=G\left(\Gamma_{N}, S_{N}\right)$ to be the Cayley graph of $\Gamma_{N}$ with respect to this generating set and $G_{N}^{\prime}=\Gamma_{N} /\langle U\rangle$ to be the quotient obtained by collapsing the $N$-cycles generated by powers of $U$. Then $\pi_{\mathbb{Z}_{N}} \cong G_{R}^{\prime}$. We now prove the upper bound of Theorem 3. For $A, B \subset V(G)$ we denote the set of edges from $A$ to $B$ by $E(A, B)$.

For $G=\pi_{\mathbb{Z}_{N}}$ we have $|R|=N$ and $\left|R^{\times}\right|=\phi(N)$ where $\phi$ is Euler's totient function. We also have the formula $\left|\Gamma_{N}\right|=\left(N^{3} / 2\right) \prod_{p \mid N}\left(1-1 / p^{2}\right)$, as shown in [10]. It follows that

$$
\left|V\left(\pi_{\mathbb{Z}_{N}}\right)\right|=\frac{N^{2}}{2} \prod_{p \mid N}\left(1-\frac{1}{p^{2}}\right) .
$$

Further, $\pi_{\mathbb{Z}_{N}}$ is regular of degree $N$.

Let $(\alpha, \beta) \in V\left(\pi_{\mathbb{Z}_{N}}\right)$. By Lemma 2 and Lemma 3, the number of vertices of $\pi_{\mathbb{Z}_{N}}$ connected to $(\alpha, \beta)$ by 2 paths of length 2 is

$$
\frac{|R|\left|R^{\times}\right|}{2}=\frac{N \phi(N)}{2}=\frac{N^{2}}{2} \prod_{p \mid N}\left(1-\frac{1}{p}\right) .
$$

Given our definitions of $n$ and $m$ from Section 1, this last number is equal to $n-m$. From (3) we obtain

$$
n-m=\frac{N^{2}}{2} \prod_{p \mid N}\left(1-\frac{1}{p^{2}}\right)-m=\frac{N^{2}}{2} \prod_{p \mid N}\left(1-\frac{1}{p}\right) .
$$

It follows that

$$
m=\frac{N^{2}}{2} \prod_{p \mid N}\left(1-\frac{1}{p}\right)\left(\prod_{p \mid N}\left(1+\frac{1}{p}\right)-1\right) .
$$

For $\alpha \in \mathbb{Z}_{N}^{\times}$let $H_{\alpha}$ denote the subgraph induced by $\{(0, \alpha)\} \cup\left\{\left(\alpha^{-1}, \beta\right) \mid \beta \in \mathbb{Z}_{N}\right\}$. It is easily shown that given $\alpha, \alpha^{\prime} \in \mathbb{Z}_{N}^{\times}$we have that $H_{\alpha}$ and $H_{\alpha}^{\prime}$ are either identical or disjoint. 
Let $\mathcal{C}_{N}$ denote the subgraph of $\pi_{\mathbb{Z}_{N}}$ induced by the set $V\left(\mathcal{C}_{N}\right)=\bigsqcup_{\alpha \in \mathbb{Z}_{N}^{\times} /\langle \pm 1\rangle} H_{\alpha}$. Since $\left|V\left(H_{\alpha}\right)\right|=N+1$ we have

$$
\left|V\left(\mathcal{C}_{N}\right)\right|=\frac{\phi(N)}{2}(N+1) .
$$

Let $\mathcal{O}_{N}$ be the subgraph in $\pi_{\mathbb{Z}_{N}}$ induced by the vertex set $\{(z, \beta) \mid(z, N) \neq 1,(z, \beta) \in$ $\left.\pi_{\mathbb{Z}_{N}}\right\}$. It is clear that $V\left(\pi_{\mathbb{Z}_{N}}\right)=V\left(\mathcal{O}_{N}\right) \sqcup V\left(\mathcal{C}_{N}\right)$. It follows that we have

$$
\left|V\left(\mathcal{O}_{N}\right)\right|=\frac{N}{2} \phi(N) \prod_{p \mid N}\left(1+\frac{1}{p}\right)-\frac{\phi(N)}{2}(N+1) .
$$

One can picture the subgraph $\mathcal{C}_{N}$ as a central "core" for $\pi_{\mathbb{Z}_{N}}$, in which the highly connected $H_{\alpha}$ 's are arranged in the form of a complete multigraph. The vertices of $\mathcal{O}_{N}$ "orbit" this core (hence our choice of $\mathcal{C}$ and $\mathcal{O}$ for notation).

Note that $\left(\alpha^{-1}, \beta\right) \in H_{\alpha}$ is adjacent to $\left(\alpha^{\prime-1}, x\right) \in H_{\alpha^{\prime}}$ if and only if $x \equiv \alpha\left(\alpha^{\prime-1} \beta \pm 1\right)$ $(\bmod N)$. It follows that there are 2 edges from $\left(\alpha^{-1}, \beta\right) \in H_{\alpha}$ to vertices in $H_{\alpha^{\prime}}$ for every $\alpha \in \mathbb{Z}_{N}^{\times} /\langle \pm 1\rangle$. Therefore, if $H_{\alpha}$ and $H_{\alpha}^{\prime}$ are distinct, then there are $2 N$ edges with one endpoint in $H_{\alpha}$ and the other in $H_{\alpha}^{\prime}$. Since $\mathcal{C}_{N}$ consists of $\phi(N) / 2$ copies of $H_{\alpha}$, this accounts for $\left(\begin{array}{c}\phi(N) / 2 \\ 2\end{array}\right) 2 N$ edges. Since $\left|E\left(H_{\alpha}\right)\right|=2 N$ we conclude that

$$
\left|E\left(\mathcal{C}_{N}\right)\right|=\left(\begin{array}{c}
\phi(N) / 2 \\
2
\end{array}\right) 2 N+2 N \frac{\phi(N)}{2}=\frac{N \phi(N)}{4}(\phi(N)+2) .
$$

The number of vertices in $\mathcal{C}_{N}$ that are of the form $v=\left(\alpha^{-1}, \beta\right)$ with $\alpha \in \mathbb{Z}_{N}^{\times}$is $N \phi(N) / 2$. For any copy of $H_{\alpha^{\prime}}$ not containing $v$ in $\mathcal{C}_{N}$, there are two edges connecting $v$ with vertices in $H_{\alpha^{\prime}}$. This gives $2\left(\frac{\phi(N)}{2}-1\right)=\phi(N)-2$ edges connecting $v$ to vertices in other copies of $H_{\alpha}$. As $v$ is adjacent to 3 other vertices in $H_{\alpha}$ and every vertex has degree $N$, we find a total of $N-\phi(N)-1$ edges connecting $v$ with vertices in $\mathcal{O}_{N}$. It follows that the number of edges with one endpoint in $\mathcal{C}_{N}$ and the other in $\mathcal{O}_{N}$ is given by

$$
\left|E\left(\mathcal{C}_{N}, \mathcal{O}_{N}\right)\right|=\frac{N \phi(N)}{2}(N-\phi(N)-1) .
$$

It is a further consequence of Lemma 2 that if $\alpha$ is such that $v \in H_{\alpha}$, then $v$ is adjacent to three vertices within $H_{\alpha}$. This gives a total of $\phi(N)+1$ edges connecting $v$ to other vertices within $\mathcal{C}_{\mathcal{N}}$.

Note that

$$
\left|E\left(\pi_{\mathbb{Z}_{N}}\right)\right|=\frac{N^{3}}{4} \prod_{p \mid N}\left(1-\frac{1}{p^{2}}\right)=\frac{N^{2}}{4} \phi(N) \prod_{p \mid N}\left(1+\frac{1}{p}\right) .
$$


Thus from (7) and (8) we have

$$
\begin{aligned}
\left|E\left(\mathcal{O}_{N}\right)\right| & =\left|E\left(\pi_{\mathbb{Z}_{N}}\right)\right|-\left|E\left(\mathcal{C}_{N}\right)\right|-\left|E\left(\mathcal{C}_{N}, \mathcal{O}_{N}\right)\right| \\
& =\frac{N^{2} \phi(N)}{4} \prod_{p \mid N}\left(1+\frac{1}{p}\right)-\frac{N \phi(N)}{4}(\phi(N)+2)-\frac{N \phi(N)}{2}(N-\phi(N)-1) \\
& =\frac{N \phi(N)}{4}\left(N \prod_{p \mid N}\left(1+\frac{1}{p}\right)+\phi(N)-2 N\right) .
\end{aligned}
$$

Note that the subgraph induced by $\mathcal{C}_{N}$ has the structure of the complete multigraph $K_{\phi(N) / 2}^{2 N}$ where each "vertex" is actually a copy of $H_{\alpha}$. Therefore, we can divide the copies of $H_{\alpha}$ arbitrarily into 2 sets of size $\phi(N) / 4$ and so $V\left(\mathcal{C}_{N}\right)=A_{\mathcal{C}} \sqcup B_{\mathcal{C}}$ and $\left|A_{\mathcal{C}}\right|=\left|B_{\mathcal{C}}\right|=$ $\left|V\left(\mathcal{C}_{N}\right)\right| / 2=\phi(N)(N+1) / 4$. Each copy of $H_{\alpha}$ in $A_{\mathcal{C}}$ contributes $2 N \phi(N) / 4$ edges to $\partial A_{\mathcal{C}}$. Since there are $\phi(N) / 4$ copies of $H_{\alpha}$ in $B_{\mathcal{C}}$ this gives a total of $2 N(\phi(N) / 4)^{2}$ edges in $\partial A_{\mathcal{C}}$.

Lemma 4. There exists a bipartition $V_{\mathcal{O}}=A_{\mathcal{O}} \sqcup B_{\mathcal{O}}$ satisfying

$$
\left|E\left(A_{\mathcal{O}}, B_{\mathcal{O}}\right)\right| \leq\left|E\left(\mathcal{O}_{N}\right)\right| / 2 .
$$

Proof. Assume that $\left|E\left(A_{\mathcal{O}}, B_{\mathcal{O}}\right)\right|>\left|E\left(\mathcal{O}_{N}\right)\right| / 2$ for every bipartition of $V_{\mathcal{O}}$. Let $V_{\mathcal{O}}=$ $A_{\mathcal{O}} \sqcup B_{\mathcal{O}}$ be a bipartition for which $\left|E\left(A_{\mathcal{O}}, B_{\mathcal{O}}\right)\right|$ is minimal. We will also use the notation $A_{\mathcal{O}}$ and $B_{\mathcal{O}}$ to denote the subgraphs induced by the sets of vertices in our bipartition.

It is an immediate consequence of our determinant criterion for adjacency that there must be vertices in $V_{\mathcal{O}}$ that are not adjacent to any other vertices in $V_{\mathcal{O}}$. Denote the set of all such vertices by $S$. The elements of $S$ have degree zero in the induced graphs $A_{\mathcal{O}}$ and $B_{\mathcal{O}}$.

There must be at least one vertex in $V_{\mathcal{O}}$ with the property that more than half its edges are in $E\left(A_{\mathcal{O}}, B_{\mathcal{O}}\right)$. This follows from $S \neq \emptyset$ and our assumptions that $\left|E\left(A_{\mathcal{O}}, B_{\mathcal{O}}\right)\right|>$ $\left|E\left(\mathcal{O}_{N}\right)\right| / 2$ and $\left|A_{\mathcal{O}}\right|=\left|B_{\mathcal{O}}\right|$. Without loss of generality we can assume that there is such a vertex $v$ in $A_{\mathcal{O}}$.

If there is a vertex $w \in S \cap B_{\mathcal{O}}$ then we could switch $v$ and $w$ to get a new decomposition with a smaller value for $\left|E\left(A_{\mathcal{O}}, B_{\mathcal{O}}\right)\right|$, thus contradicting the minimality of our decomposition. It follows that $S \subset A_{\mathcal{O}}$. Further, all of the vertices in $B_{\mathcal{O}}$ must have at most the same number of edges incident with $A_{\mathcal{O}}$ as with other vertices in $B_{\mathcal{O}}$. A vertex in $B_{\mathcal{O}}$ not satisfying this condition could be switched with a vertex in $S$ to get a new decomposition, contradicting our minimality assumption.

For $v \in A_{\mathcal{O}}$ let $v_{+}$denote the edges of $v$ incident with an edge in $E\left(A_{\mathcal{O}}, B_{\mathcal{O}}\right)$ and let $v_{-}$denote the other edges incident with $v$. For $w \in B_{\mathcal{O}}$ let $w_{-}$be the edges of $w$ incident with an edge in $E\left(A_{\mathcal{O}}, B_{\mathcal{O}}\right)$ and $w_{+}$the remaining edges of $w$. Now, assume for a contradiction that

$$
\sum_{v \in A_{\mathcal{O}}} v_{+}-v_{-}<\sum_{w \in B_{\mathcal{O}}} w_{+}-w_{-}
$$


We have that

$$
\sum_{v \in A_{\mathcal{O}}} v_{+}-v_{-}=\sum_{v \in A_{\mathcal{O}}} v_{+}-\sum_{v \in A_{\mathcal{O}}} v_{-}=\left|E\left(A_{\mathcal{O}}, B_{\mathcal{O}}\right)\right|-2\left|E\left(A_{\mathcal{O}}\right)\right|
$$

and

$$
\sum_{w \in B_{\mathcal{O}}} w_{+}-w_{-}=\sum_{w \in B_{\mathcal{O}}} w_{+}-\sum_{w \in B_{\mathcal{O}}} w_{-}=2\left|E\left(B_{\mathcal{O}}\right)\right|-\left|E\left(A_{\mathcal{O}}, B_{\mathcal{O}}\right)\right|
$$

It follows that

$$
\left|E\left(A_{\mathcal{O}}, B_{\mathcal{O}}\right)\right|-2\left|E\left(A_{\mathcal{O}}\right)\right|<2\left|E\left(B_{\mathcal{O}}\right)\right|-\left|E\left(A_{\mathcal{O}}, B_{\mathcal{O}}\right)\right|
$$

which implies that $\left|E\left(A_{\mathcal{O}}, B_{\mathcal{O}}\right)\right|<\left|E\left(A_{\mathcal{O}}\right)\right|+\left|E\left(B_{\mathcal{O}}\right)\right|$. However, since $\left|E\left(A_{\mathcal{O}}, B_{\mathcal{O}}\right)\right|>$ $\left|E\left(\mathcal{O}_{N}\right)\right| / 2$ this gives

$$
\left|E\left(\mathcal{O}_{N}\right)\right|=\left|E\left(A_{\mathcal{O}}, B_{\mathcal{O}}\right)\right|+\left|E\left(A_{\mathcal{O}}\right)\right|+\left|E\left(B_{\mathcal{O}}\right)\right|>\frac{\left|E\left(\mathcal{O}_{N}\right)\right|}{2}+\frac{\left|E\left(\mathcal{O}_{N}\right)\right|}{2}
$$

which is a contradiction. Therefore we must have

$$
\sum_{v \in A_{\mathcal{O}}} v_{+}-v_{-} \geq \sum_{w \in B_{\mathcal{O}}} w_{+}-w_{-}
$$

As $\emptyset \neq S \subset A_{\mathcal{O}}$, there must be some $v \in A_{\mathcal{O}}$ and some $w \in B_{\mathcal{O}}$ for which $v_{+}-v_{-}>$ $w_{+}-w_{-}$. If we switch $v$ to $B_{\mathcal{O}}$ and $w$ to $A_{\mathcal{O}}$ then we get a new bipartition $A_{\mathcal{O}}^{\prime}, B_{\mathcal{O}}^{\prime}$ with $\left|E\left(A_{\mathcal{O}}^{\prime}, B_{\mathcal{O}}^{\prime}\right)\right|<\left|E\left(A_{\mathcal{O}}, B_{\mathcal{O}}\right)\right|$, again contradicting our minimality condition. It follows that there must be some bipartition satisfying $\left|E\left(A_{\mathcal{O}}, B_{\mathcal{O}}\right)\right| \leq\left|E\left(\mathcal{O}_{N}\right)\right| / 2$, as was to be shown.

Lemma 4 assures us that we can decompose $V\left(\mathcal{O}_{N}\right)=A_{\mathcal{O}} \sqcup B_{\mathcal{O}}$ in such a way that

$$
\left|A_{\mathcal{O}}\right|=\left|B_{\mathcal{O}}\right|=\frac{N \phi(N)}{4} \prod_{p \mid N}\left(1+\frac{1}{p}\right)-\frac{(N+1) \phi(N)}{4}
$$

and so that $\left|E\left(A_{\mathcal{O}}, B_{\mathcal{O}}\right)\right| \leq\left|E\left(\mathcal{O}_{N}\right)\right| / 2$.

Now

$$
\left|E\left(\mathcal{C}_{N}, \mathcal{O}_{N}\right)\right|=\left|E\left(A_{\mathcal{O}}, A_{\mathcal{C}}\right)\right|+\left|E\left(A_{\mathcal{O}}, B_{\mathcal{C}}\right)\right|+\left|E\left(B_{\mathcal{O}}, A_{\mathcal{C}}\right)\right|+\left|E\left(B_{\mathcal{O}}, B_{\mathcal{C}}\right)\right|
$$

and it follows that one of $\left|E\left(A_{\mathcal{O}}, A_{\mathcal{C}}\right)\right|+\left|E\left(B_{\mathcal{O}}, B_{\mathcal{C}}\right)\right|$ or $\left|E\left(A_{\mathcal{O}}, B_{\mathcal{C}}\right)\right|+\left|E\left(B_{\mathcal{O}}, A_{\mathcal{C}}\right)\right|$ must be less than or equal to $\frac{1}{2}\left|E\left(\mathcal{C}_{N}, \mathcal{O}_{N}\right)\right|$. Thus one of the sets $A_{\mathcal{O}} \sqcup A_{\mathcal{C}}$ or $A_{\mathcal{O}} \sqcup B_{\mathcal{C}}$ must 
have boundary less than or equal to

$$
\begin{aligned}
\frac{1}{2}\left|E\left(\mathcal{C}_{N}, \mathcal{O}_{N}\right)\right| & +\frac{1}{2}\left|E\left(\mathcal{O}_{N}\right)\right|+2 N\left(\frac{\phi(N)}{4}\right)^{2} \\
= & \frac{N \phi(N)}{4}(N-\phi(N)-1) \\
& \quad+\frac{N \phi(N)}{8}\left(N \prod_{p \mid N}\left(1+\frac{1}{p}\right)+\phi(N)-2 N\right)+\frac{N \phi(N)^{2}}{8} \\
& =\frac{N \phi(N)}{4}\left(\frac{N}{2} \prod_{p \mid N}\left(1+\frac{1}{p}\right)-1\right)
\end{aligned}
$$

where we applied (8) and (9). From (5) and (6), the number of vertices in each of the sets above is

$$
\frac{\left|V\left(\mathcal{C}_{N}\right)\right|}{2}+\frac{\left|V\left(\mathcal{O}_{N}\right)\right|}{2}=\frac{N \phi(N)}{4} \prod_{p \mid N}\left(1+\frac{1}{p}\right) .
$$

Thus we have

$$
h\left(\pi_{\mathbb{Z}_{N}}\right) \leq \frac{\frac{N \phi(N)}{4}\left(\frac{N}{2} \prod_{p \mid N}\left(1+\frac{1}{p}\right)-1\right)}{\frac{N \phi(N)}{4} \prod_{p \mid N}\left(1+\frac{1}{p}\right)}=\frac{N}{2}-\frac{1}{\prod_{p \mid N}\left(1+\frac{1}{p}\right)} .
$$

This proves the upper bound of (i) of Theorem 3. To prove the lower bound of (i) note that by (4) the condition $k^{2} \geq r(n-m)$ applied to $\pi_{\mathbb{Z}_{N}}$ gives $1 \geq \prod_{p \mid N}\left(1-\frac{1}{p}\right)$ which always holds. The result then follows from a direct application of (i) Theorem 1.

Let $\{N\}$ denote an increasing sequence such that $\prod_{p \mid N}\left(1+\frac{1}{p}\right) \rightarrow 1$ as $N \rightarrow \infty$. Then for any $\epsilon>0$ we have

$$
\sqrt{1-2 \prod_{p \mid N}\left(1-\frac{1}{p}\right)+\prod_{p \mid N}\left(1-\frac{1}{p^{2}}\right)}<\epsilon
$$

for sufficiently large $N$. This result and a direct application of the upper and lower bounds from $(i)$ of Theorem 3 gives the rest of part (i).

We have

$$
\frac{\left|E\left(\mathcal{O}_{N}\right)\right|}{\left|V\left(\mathcal{O}_{N}\right)\right|}=\frac{N}{2}\left(\frac{\prod_{p \mid N}\left(1+\frac{1}{p}\right)+\prod_{p \mid N}\left(1-\frac{1}{p}\right)-2}{\prod_{p \mid N}\left(1+\frac{1}{p}\right)-1-\frac{1}{N}}\right)=\frac{N}{2} C_{N} .
$$

For $\epsilon>0$ let the primes $p \mid N$ be large enough so that $1 / \prod_{p \mid N}\left(1+\frac{1}{p}\right)<\epsilon$. Let $\epsilon(N)$ denote any function that goes to 0 as $N \rightarrow \infty$. From Corollary 3.1 in [6] there exists some $A_{\mathcal{O}} \subseteq V\left(\mathcal{O}_{N}\right)$ with $\left|A_{\mathcal{O}}\right|=\left|V\left(\mathcal{O}_{N}\right)\right| / 2$ and so that

$$
\left|\partial A_{\mathcal{O}}\right|=\frac{N}{2} C_{N}\left|A_{\mathcal{O}}\right|+\epsilon(N)\left|A_{\mathcal{O}}\right| .
$$


Therefore, as in part (i) we have

$$
\begin{aligned}
h\left(\pi_{\mathbb{Z}_{N}}\right) \leq & \frac{\frac{N \phi(N)}{4}(n-\phi(N)-1)+\frac{N}{2} c_{N} \frac{\left|V\left(\mathcal{O}_{N}\right)\right|}{2}+\epsilon(N) \frac{\left|V\left(\mathcal{O}_{N}\right)\right|}{2}+\frac{N \phi(N)^{2}}{8}}{\frac{N \phi(N)}{4} \prod_{p \mid N}\left(1+\frac{1}{p}\right)} \\
= & \frac{N}{2} C_{N}\left(\frac{\prod_{p \mid N}\left(1+\frac{1}{p}\right)-1-\frac{1}{N}}{\prod_{p \mid N}\left(1+\frac{1}{p}\right)}\right)+\frac{N}{\prod_{p \mid N}\left(1+\frac{1}{p}\right)}-\frac{\phi(N)}{2 \prod_{p \mid N}\left(1+\frac{1}{p}\right)} \\
& +\epsilon(N) \frac{\prod_{p N}\left(1+\frac{1}{p}\right)-1-\frac{1}{N}}{\prod_{p \mid N}\left(1+\frac{1}{p}\right)} \\
& \leq \frac{N}{2} C(N)+\epsilon N-\frac{\epsilon \phi(N)}{2}+\epsilon(N) \frac{\prod_{p \mid N}\left(1+\frac{1}{p}\right)-1-\frac{1}{N}}{\prod_{p \mid N}\left(1+\frac{1}{p}\right)}
\end{aligned}
$$

where

$$
C(N)=\frac{\prod_{p \mid N}\left(1+\frac{1}{p}\right)+\prod_{p \mid N}\left(1-\frac{1}{p}\right)-2}{\prod_{p \mid N}\left(1+\frac{1}{p}\right)}
$$

and $0<C(N)<1$. This proves the first part of (ii).

To show that $\pi_{\mathbb{Z}_{N}}$ is not Ramanujan it suffices to show that $h\left(\pi_{\mathbb{Z}_{N}}\right)$ is sufficiently small with respect to the degree. In [13] it was shown that for $R=\mathbb{Z}_{p^{r}}$ with prime $p \equiv 1$ $(\bmod 4)$ we have

$$
\frac{p^{r}(p-1)}{2(p+1)} \geq h\left(\pi_{R}\right)
$$

Since $h(G) \geq\left(k-\lambda_{1}\right) / 2$ from Theorem 1.2.3 of [7], for example, we have $p^{r}(p-1) /(p+1)>$ $p^{r}-\lambda_{1}$. It follows that if

$$
\frac{p^{2 r}}{p^{r}-1} \geq(p+1)^{2}
$$

then $\pi_{R}$ is not Ramanujan. It is easy to see that this holds for $r, p \geq 3$. From [7] and (10), for sufficiently large odd composite $N$ there is some $c<\frac{1}{2}$ so that $c N>\frac{N-\lambda_{1}}{2}$. Thus $\lambda_{1}>N(1-2 c) \geq 2 \sqrt{N-1}$ for such $N$. This proves (ii) of Theorem 3 .

\section{Applications to Arithmetic Riemann Surfaces}

Recall that the group $\Gamma_{N}$ acts on the complex upper half plane $\mathcal{H}=\{z=x+i y \mid y>0\}$ via linear fractional transformations. Let $\mathcal{F}_{N}$ denote a fundamental domain for this action. 
It is possible to construct $\mathcal{F}_{N}$ so that $\mathcal{F}_{N}$ consists of copies of

$$
\begin{aligned}
& \mathcal{F}_{1}=\{z=x+i y\left.\in \mathcal{H}|| z \mid>1,-\frac{1}{2} \leq x<\frac{1}{2}\right\} \\
& \bigsqcup\left\{z=x+i y \in \mathcal{H}|| z \mid=1,-\frac{1}{2} \leq x \leq 0\right\}
\end{aligned}
$$

that do not overlap. That is, one can consider that copies of $\mathcal{F}_{1}$ tile $\mathcal{F}_{N}$. Note that $\mathcal{F}_{N}$ can be viewed as a Riemann surface, and we denote this surface by $\Gamma_{N} \backslash \mathcal{H}$.

We can associate to $\Gamma_{N} \backslash \mathcal{H}$ a graph whose vertices are the copies of the tiles $\mathcal{F}_{1}$. Two vertices are connected by an edge if and only if the respective tiles share a boundary. The graphs constructed in this manner are isomorphic to the Cayley graphs of $P S L_{2}\left(\mathbb{Z}_{N}\right)$ with respect to the generators that define the fundamental domains. (An explicit isomorphism is shown in [20].)

The Cheeger constant of a closed, compact Riemannian manifold $M$ is defined by

$$
h(M)=\inf _{S} \frac{\operatorname{area}(S)}{\min (\operatorname{vol}(A), \operatorname{vol}(B))}
$$

where $S$ runs over all hypersurfaces that divide $M$ into disjoint pieces $A$ and $B$. The isoperimetric number of a graph is a discrete version of the Cheeger constant of a manifold. Upper bounds on the isoperimetric numbers of the Cayley graphs $G_{N}$ associated to $\Gamma_{N} \backslash \mathcal{H}$ immediately give upper bounds on the Cheeger constants of $\Gamma_{N} \backslash \mathcal{H}$. In fact, Buser [4] introduced the discrete version of $h(M)$ to study the Cheeger constants of these manifolds.

More precisely, if $A \subset V\left(G_{N}\right)$ then every edge in $\partial A$ represents a boundary edge of a fundamental domain. Since each such edge of the fundamental domain has length $\log (3)$, see [5] for example, and a fundamental domain has area $\pi / 3$ this gives

$$
h\left(\Gamma_{N} \backslash \mathcal{H}\right) \leq \frac{3 \log (3)}{\pi} h\left(G_{N}\right) .
$$

In [5] this estimate (for $N$ a prime congruent to 1 modulo 4) was used to show that this discrete approach would be ineffective to tackle Selberg's eigenvalue conjecture. In particular, they showed that for such $N, h\left(\Gamma_{N} \backslash \mathcal{H}\right) \leq .5245 \ldots$ and hence was too small to improve known bounds on the smallest eigenvalue of the Laplacian on $\Gamma_{N} \backslash \mathcal{H}$. From Section 3 we have that $h\left(G_{N}\right) \leq h\left(\pi_{\mathbb{Z}_{N}}\right) / N$. Combining this with the upper bound on $h\left(\pi_{\mathbb{Z}_{N}}\right)$ from (i) of Theorem 3 gives $h\left(\Gamma_{N} \backslash \mathcal{H}\right) \leq 0.5245$ for all $N$. Furthermore, from the probabilistic methods of [6] we have the asymptotic result that, for sufficiently large $N$,

$$
h\left(\Gamma_{N} \backslash \mathcal{H}\right) \leq \frac{3}{8 \pi}\left(\operatorname{arccosh}(3)+2 \operatorname{arccosh}\left(\frac{3}{2}\right)\right) h\left(G_{N}\right) \approx 0.4402 \ldots
$$

From a direct computation using (ii) of Theorem 3 we can easily show that for sufficiently large $N$ and $\prod_{p \mid N}\left(1+\frac{1}{p}\right)$ sufficiently large that

$$
h\left(\Gamma_{N} \backslash \mathcal{H}\right) \leq C(N) \cdot 0.4402=A
$$

where $C(N)$ is as in (11) in the proof of (ii) of Theorem 3 in Section 4 . This proves Corollary 2. 


\section{References}

[1] N. Alon, O. Schwartz, A. Shapira, An elementary construction of constant-degree expanders, Combin. Probab. and Comput. 17 (2008), 319-327.

[2] L. Babai, Spectra of Cayley Graphs, J. Combin. Theory Ser. B, 27 (1979), 180-189.

[3] B. Bollobás, The isoperimetric number of random regular graphs, European J. Combin. 9 (1988), 241-244.

[4] P. Buser, Cubic graphs and the first eigenvalue of a Riemann surface, Math. Z. 162 (1978), 87-99.

[5] R. Brooks, P. Perry, P. Petersen, On Cheeger's inequality, Comment. Math. Helv. 68 (1993), 599-621.

[6] R. Brooks, A. Zuk, On the asymptotic isoperimetric constants for Riemann surfaces and graphs, J. Differential Geom. 62 (2002), 49-78.

[7] G. Davidoff, P. Sarnak, A. Valette, Elementary Number Theory, Group Theory, and Ramanujan Graphs, London Math. Soc. Student Texts 55, Cambridge Univ. Press, Cambridge, UK, 2003.

[8] M. DeDeo, D. Lanphier, M. Minei, The spectrum of Platonic graphs over finite fields, Discrete Math. 307 (2007), 1074-1081.

[9] P.E. Gunnells, Some elementary Ramanujan graphs, Geom. Dedicata, 112 (2005), 51-63.

[10] S. Katok, Fuchsian Groups, Chicago Lectures in Mathematics, The University of Chicago Press, Chicago, 1992.

[11] M. Krivelevich, B. Sudakov, V.H. Vu, N.C. Wormald, Random regular graphs of high degree, Random Structures Algorithms 18(2001), 346-363.

[12] P. Kutzko, The characters of the binary modular congruence group, Bull. Amer. Math. Soc. (N.S.) 79, (1973), 702-704.

[13] D. Lanphier, J. Rosenhouse, Cheeger constants of Platonic graphs, Discrete Math. 277 (2004), 101-113.

[14] D. Lanphier, J. Rosenhouse, Lower bounds on the Cheeger constants of highly connected regular graphs, Congr. Numer. 173 (2005), 65-74.

[15] W. Li, Y. Meemark, Ramanujan graphs on cosets of $P G L_{2}\left(\mathbb{F}_{q}\right)$, Finite Fields Appl. 11 (2005), 511-543.

[16] A. Lubotzky, Discrete groups, expanding graphs, and invariant measures, with an appendix by J. Rogawski, Progr. in Math. 125, Birkhäuser Verlag, Basel, CH, 1994.

[17] B. Mohar, Isoperimetric numbers of graphs, J. Combin. Theory Ser. B 47 (1989), 274-291.

[18] I.I. Piatetski-Shapiro, Complex representations of $G L(2, K)$ for finite fields $K$, Contemp. Math. 16, 1983.

[19] O. Reingold, S. Vadhan, A. Wigderson, Entropy waves, the zig-zag graph product, and new constant-degree expanders, Ann. of Math. 155 (2002), 157-187.

[20] J. Rosenhouse, Constructing Cayley Graphs Via Tesselations of Riemann Surfaces, Congr. Numer., 179 (2006), 209-213. 